\title{
Effect of Head Position and Tube Entry on Corneal Endothelial Cells in Patients with Glaucoma Drainage Implants: A Cross- sectional Study
}

\author{
Jungbin Han, Chungkwon Yoo, Ji-Hye Park, Yong Yeon Kim \\ Department of Ophthalmology, Korea University College of Medicine, Seoul, Korea
}

Purpose: To investigate the effect of head tilt on the tube position of the Ahmed glaucoma valve (AGV) implanted in patients with glaucoma and to assess how the head tilt-induced alterations of tube parameters and the level of tube entry influence corneal endothelial cell density (ECD).

Methods: A total of 29 eyes of 26 patients with AGV implantation were included. Tube-cornea distance, tube-cornea angle, and intracameral tube length were measured using anterior segment optical coherence tomography in three different head positions (neutral, $30^{\circ}$ temporalward tilt, and $30^{\circ}$ nasalward tilt). The tube entry was assessed using static gonioscopy. ECD was measured using specular microscopy before and after surgery.

Results: The mean tube-cornea distance, tube-cornea angle, and intracameral tube length (neutral: $0.87 \pm 0.39 \mathrm{~mm}, 30.56 \pm$ $5.89^{\circ}$, and $3.10 \pm 0.82 \mathrm{~mm}$, respectively) decreased with head tilts (temporalward: $0.82 \pm 0.39 \mathrm{~mm}, 29.27 \pm 5.82^{\circ}$, and $3.04 \pm$ $0.82 \mathrm{~mm}$, respectively; nasalward: $0.83 \pm 0.40 \mathrm{~mm}, 29.61 \pm 6.04^{\circ}$, and $3.05 \pm 0.81 \mathrm{~mm}$, respectively; all $p<0.01$ ). The multivariate analyses found age and the tube insertion level to be associated with postoperative changes in the central ECD $(p=0.039$ and 0.013 , respectively), and the postoperative follow-up period and tube insertion level to be associated with the difference between the inferonasal and superotemporal ECDs ( $p=0.034$ and 0.007 , respectively).

Conclusions: Mild alterations of head positions induced changes in the intracameral tube positions of AGV implants; nevertheless, it did not significantly affect ECD loss. However, the eyes with tubes inserted anteriorly to Schwalbe's line may be more susceptible to corneal ECD loss.

Key Words: Corneal endothelial cell loss, Glaucoma, Glaucoma drainage implants, Optical coherence tomography, Posture

Received: August 14, 2020 Final revision: September 9, 2020

Accepted: September 21, 2020

Corresponding Author: Chungkwon Yoo, MD, PhD. Department of Ophthalmology, Korea University College of Medicine, 73 Inchon-ro, Seongbuk-gu, Seoul 02841, Korea. Tel: 82-2-920-5521, Fax: 82-2-9246820, E-mail: ckyoomd@korea.ac.kr

This study was presented at the 121st annual meeting of the Korean Ophthalmological Society on April 5-7, 2019 in Busan, Korea
Currently, reduction of intraocular pressure (IOP) is the only proven treatment for glaucoma [1]. IOP reduction can be achieved with medication, laser, or surgery. Surgical treatment for glaucoma includes trabeculectomy, glaucoma drainage device (GDD) implantation, trabecular bypass stents, ab interno trabeculectomy, and subconjunctival stenting [2-6]. The emergence of minimally invasive glaucoma surgery has broadened the scope of surgical alterna- 
tives for glaucoma treatment. However, several minimally invasive glaucoma surgeries are indicated for early to moderate open-angle glaucoma because of their limited IOP-reducing efficacy and lack of long-term data when compared with conventional glaucoma surgeries, such as trabeculectomy or GDD implantation [6].

Generally, GDD implantation is indicated for advanced or refractory glaucoma. However, recent randomized trials revealed its efficacy and survival are comparable to trabeculectomy $[7,8]$. The United States medical insurance claims data demonstrated a marked increase in GDD implantation over the last decade [9]. Despite its continuous growth, postoperative complications, including hypotony, conjunctival erosion, cataract, and endophthalmitis, may occur following GDD surgery [10].

Among the possible postoperative complications, the most alarming is corneal decompensation, with an incidence of $3.3-30 \%$ in patients with long-term follow-up periods [11,12]. Although the exact mechanism of corneal endothelial cell damage remains unclear, intermittent tube-corneal touch, turbulence at the tip of the silicon tube, anterior chamber inflammation, and tube-uveal touch may be the possible contributors $[12,13]$.

Anterior segment optical coherence tomography (ASOCT) imaging provides in vivo visualization of the drainage tube in the eye. A few studies showed that corneal endothelial cell damages are related to the distance from the tip of the tube to the cornea $[14,15]$. However, patients with a continuous decrease in corneal endothelial cell number are often observed, despite good intracameral location of the tube. Moreover, a study showed that the location and length of the tube are not significantly related with the corneal endothelial cell loss [16]. Recently, our group investigated the effects of the lateral decubitus position on the anterior chamber angle in healthy subjects and demonstrated that anterior chamber angle parameters were altered by postural shift from sitting to the lateral decubitus position [17]. In this context, we hypothesized that the postoperative corneal endothelial cell damage may be related to possible alterations in tube parameters induced by different head positions, occurring in our daily life.

Therefore, we conducted the present study to investigate the effect of head tilt on tube position of the Ahmed glaucoma valve (AGV) using AS-OCT in patients with glaucoma and to assess how the head tilt-induced alterations of the tube parameters and level of tube entry influence corneal endothelial cell density (ECD).

\section{Materials and Methods}

This was an observational, cross-sectional study conducted from July 2018 to January 2019 at Korea University Anam Hospital. This prospective study to evaluate effect of head position and tube entry on corneal endothelial cells in patients with GDD implants was approved by the institutional review board and ethics committee of Korea University Anam Hospital (2018AN0273) and was performed according to the tenets of the Declaration of Helsinki. Written informed consent to participate in the research protocol was obtained from all subjects. This study included patients who underwent the AGV valve implantation surgery in the superotemporal quadrant and had been followed up for a minimum of three months after surgery. All patients in this study had refractory glaucoma and did not respond to maximal medical treatment and/or previous trabeculectomy procedures. Patients with preexisting corneal pathology, previous corneal grafts, or history of any intraocular surgery after AGV implantation were excluded.

IOP was measured using Goldmann applanation tonometry. Axial length and anterior chamber depth were obtained using the IOL master (Carl Zeiss Meditec, Jena, Germany). Central corneal thickness (CCT), coefficient of variation $(\mathrm{CV})$, and $\mathrm{ECD}$ were measured using the noncontact-type specular microscope (SP-2000P; Topcon America Corp., Paramus, NJ, USA). Static gonioscopy was performed to assess the level of tube insertion in the anterior chamber angle by a single glaucoma specialist (CY). Tube-related parameters were assessed from the anterior chamber angle images obtained with AS-OCT (Visante OCT; Carl Zeiss Meditec, Dublin, CA, USA).

\section{AS-OCT scanning in different head positions}

AGV tube position was measured using AS-OCT with the anterior segment scan protocol by a single examiner after aligning the scan line to the axis of the AGV tube. AGV tube position measurements were performed in the neutral, $30^{\circ}$ temporalward tilt, and $30^{\circ}$ nasalward tilt positions of the head. The neutral head position was defined as the individual's head being in the straight upright position 
without any head tilt or turn. Temporalward tilt position was defined as tilting the individual's head along the naso-occipital axis toward the temporalward shoulder of the study eye, and nasalward tilt position was defined as tilting the individual's head along the naso-occipital axis toward the nasalward shoulder of the study eye. Degree of tilting was confirmed with the level meter. All measurements were performed in a dark room to minimize the effect of pupil variations.

\section{Measurement of the AGV tube position and tube in- sertion level}

Tube parameters, including the distance between the tube and cornea (TCD), the angle between tube and cornea (TCA), and the intracameral tube length (TL), were measured from the scanned images using the caliper and angle
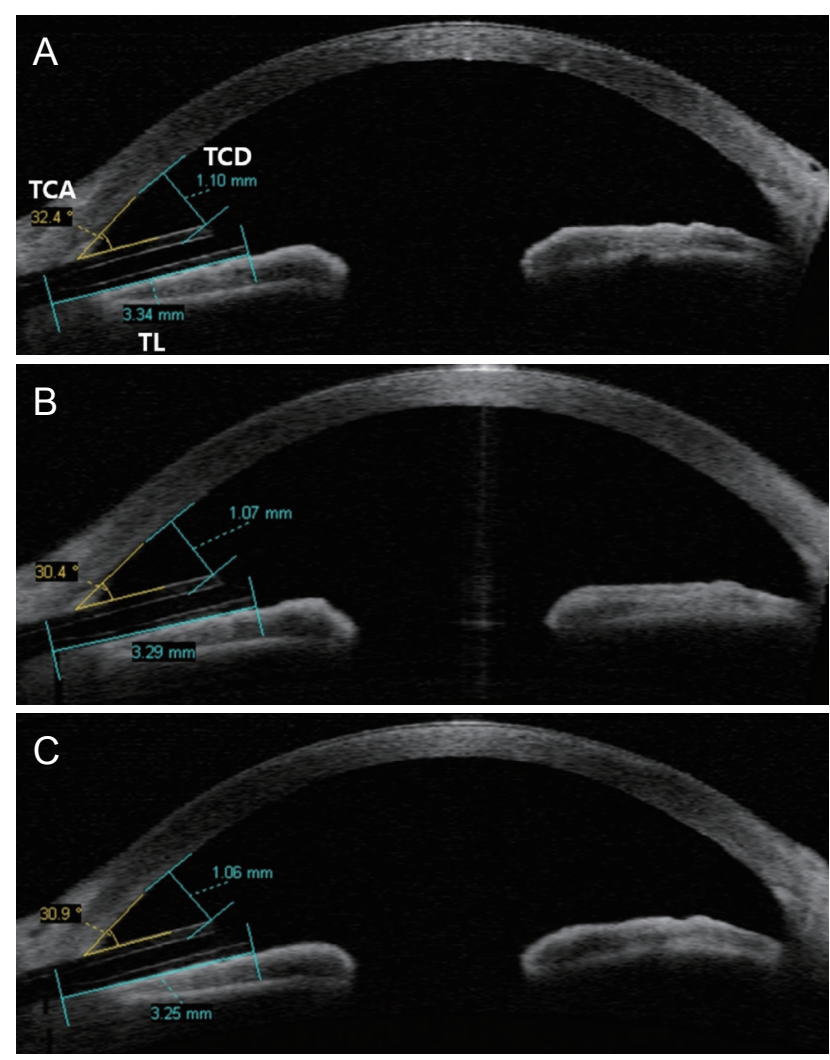

Fig. 1. Tube parameters measured from the anterior chamber image scanned using the anterior segment optical coherence tomography. (A) Anterior chamber image in neutral head position.

(B) Anterior chamber image in temporalward head tilt position. (C) Anterior chamber image in nasalward head tilt position. TCA = tube-cornea angle; $\mathrm{TCD}=$ tube-cornea distance; $\mathrm{TL}=$ tube length. tools of the device's software by the two observers in a masked fashion (Fig. 1A-1C). The mean value of the readings obtained by the two observers was used as the measurement. The ocular torsion angle was defined as the difference between the head tilt angle and AS-OCT scan line orientation of image analysis report. The level of tube insertion in the anterior chamber angle was evaluated using gonioscopy by a single glaucoma specialist, and the study eyes were divided into two groups according to the location of the tube in the anterior chamber angle relative to Schwalbe's line, that is, whether the tube entry was anterior to Schwalbe's line.

\section{Measurement of corneal ECD}

$\mathrm{ECD}, \mathrm{CV}$, and CCT were measured using the noncontact-type specular microscopy. ECD measurement was performed in the central, superotemporal, and inferonasal areas with the patients fixating on the light that was repositioned according to the area being imaged.

\section{Statistical analysis}

Statistical analyses were performed using the IBM SPSS Statistics ver. 23.0 (IBM Corp., Armonk, NY, USA). Preoperative and postoperative baseline characteristics were compared using the Wilcoxon signed-rank test with Bonferroni's correction. Postoperative ECDs were compared between different corneal areas using the Mann-Whitney $U$-test with Bonferroni's correction. Tube position parameters were compared between different head positions using the Wilcoxon signed-rank test with Bonferroni's correction. A general linear model was used to assess the factors which influence ECD. Unless stated otherwise, $p$-value $\leq 0.05$ was considered to indicate statistical significance.

\section{Results}

A total of 26 patients (29 eyes) were consecutively enrolled in this study (Table 1). Tables 1 and 2 list the demographic variables and ocular biometric parameters measured before and after AGV implantation. Mean values of IOP, ECD, and CV decreased after surgery (preoperative: $31.0 \pm 10.0 \mathrm{mmHg}$ to postoperative IOP: $10.6 \pm 3.1 \mathrm{mmHg}$, $p<0.001$; preoperative: $2,607.7 \pm 567.2$ cells $/ \mathrm{mm}^{2}$ to post- 
operative central corneal ECD: $2,113.7 \pm 587.5$ cells $/ \mathrm{mm}^{2}$, $p<0.001$; preoperative: $17.0 \pm 5.5$ to postoperative central corneal CV: $21.8 \pm 5.8, p<0.001)$. The mean postoperative ECD was higher in the central cornea than in the superotemporal cornea but lower than that in the inferonasal cornea (postoperative central corneal ECD: 2,113.7 \pm 587.5

Table 1. Demographics of the study eyes

\begin{tabular}{lc}
\hline Characteristics & Value \\
\hline Eyes & 29 \\
Age (yr) & $54.3 \pm 12.3$ \\
Sex, male & 20 \\
Laterality (right eye) & 20 \\
Lens status (phakic : pseudophakic : aphakic) & $12: 15: 2$ \\
Postoperative follow-up period (mon) & $24.9 \pm 31.3$ \\
Tube entry anterior to Schwalbe's line & 12 \\
\hline
\end{tabular}

Values are presented as number or mean \pm standard deviation. cells $/ \mathrm{mm}^{2}$, postoperative superotemporal corneal ECD: $1,646.3 \pm 793.9$ cells $/ \mathrm{mm}^{2}$, and postoperative inferonasal corneal ECD: 2,226.0 \pm 639.9 cells $/ \mathrm{mm}^{2}$ ) (Table 2).

The mean TCD decreased from $0.87 \pm 0.39$ to $0.82 \pm 0.39$ and $0.83 \pm 0.40 \mathrm{~mm}$ when the head position was changed from neutral to temporalward and nasalward tilt positions, respectively ( $p<0.001$ and 0.003 , respectively). During the same alteration of head positions, the mean TCA and TL also showed a decrease (TCA: $30.56 \pm 5.89^{\circ}$ to $29.27 \pm$ $5.82^{\circ}$ [temporalward tilt] and $29.61 \pm 6.04^{\circ}$ [nasalward tilt], both $p<0.001$; TL: $3.10 \pm 0.82$ to $3.04 \pm 0.82 \mathrm{~mm}$ [temporalward tilt] and $3.05 \pm 0.81 \mathrm{~mm}$ [nasalward tilt], $p=0.001$ and 0.003 , respectively) (Table 3). The interobserver agreement of the TCD, TCA, and TL was excellent. The intraclass correlation was $0.984(0.975-0.988), 0.974(0.960-$ $0.981)$, and 0.978 (0.966-0.984), respectively.

The mean central corneal ECD significantly decreased after surgery (preoperative: $2607.7 \pm 567.2 \mathrm{cells} / \mathrm{mm}^{2}$ to postoperative: $2,113.7 \pm 587.5$ cells $/ \mathrm{mm}^{2}, p<0.001$ ) howev-

Table 2. Comparison of preoperative and postoperative ocular biometric parameters of the study eyes

\begin{tabular}{lccc}
\hline & Preoperative & Postoperative & $p$-value \\
\hline Axial length $(\mathrm{mm})$ & $24.5 \pm 2.3$ & - & - \\
Anterior chamber depth $(\mathrm{mm})$ & $3.8 \pm 0.7$ & - & $0.005^{*}$ \\
Central corneal thickness $(\mu \mathrm{m})$ & $541.1 \pm 40.5$ & $525.7 \pm 37.1$ & $10.6 \pm 3.1$ \\
Intraocular pressure $(\mathrm{mmHg})$ & $31.0 \pm 10.0$ & $21.8 \pm 5.8$ & $<0.001^{*}$ \\
Central CV & $17.0 \pm 5.5$ & $2,113.7 \pm 587.5$ & $<0.001^{*}$ \\
Central ECD (cells $\left./ \mathrm{mm}^{2}\right)$ & $2,607.7 \pm 567.2$ & $2,226.0 \pm 639.9$ & $0.110^{\dagger}$ \\
Inferonasal ECD $\left(\mathrm{cells} / \mathrm{mm}^{2}\right)$ & - & $1,646.3 \pm 793.9$ & $0.002^{\dagger}$ \\
Superotemporal ECD $\left(\mathrm{cells} / \mathrm{mm}^{2}\right)$ & - & & \\
\hline
\end{tabular}

Values are presented as mean \pm standard deviation.

$\mathrm{CV}=$ coefficient of variation; $\mathrm{ECD}=$ endothelial cell density.

"Preoperative vs preoperative values; Wilcoxon signed-rank test with Bonferroni's correction; $p<0.0125$ is considered significant; ${ }^{\dagger}$ Versus postoperative central ECD; Mann-Whitney $U$-test with Bonferroni's correction; $p<0.025$ is considered significant.

Table 3. Tube parameters measured from images scanned using the anterior segment optical coherence tomography in different head positions

\begin{tabular}{lccccccc}
\hline Head position & TCD $(\mathrm{mm})$ & $p$-value & TCA $\left(^{\circ}\right)$ & $p$-value & TL $(\mathrm{mm})$ & $p$-value & OTA $^{*}\left(^{\circ}\right)$ \\
\hline Neutral & $0.87 \pm 0.39$ & - & $30.56 \pm 5.89$ & - & $3.10 \pm 0.82$ & - & - \\
Temporalward tilt & $0.82 \pm 0.39$ & 0.000 & $29.27 \pm 5.82$ & $<0.001$ & $3.04 \pm 0.82$ & 0.001 & $18.27 \pm 8.33$ \\
Nasalward tilt & $0.83 \pm 0.40$ & 0.003 & $29.61 \pm 6.04$ & $<0.001$ & $3.05 \pm 0.81$ & 0.003 & $21.41 \pm 7.48$ \\
\hline
\end{tabular}

Values are presented as mean \pm standard deviation.

$\mathrm{TCD}=$ tube-cornea distance; TCA = tube-cornea angle; TL = tube length; OTA = ocular torsion angle.

${ }^{*}$ Versus neutral head position; Wilcoxon signed-rank test. Because of multiple comparisons, Bonferroni's correction requires $p<0.025$ to reach a statistical significance. 
Table 4. Factors associated with changes in the central corneal endothelial cell density following Ahmed glaucoma valve implantation

\begin{tabular}{|c|c|c|c|c|}
\hline & \multicolumn{2}{|c|}{ Univariate analysis } & \multicolumn{2}{|c|}{ Multivariate analysis } \\
\hline & B coefficient & $p$-value & B coefficient & $p$-value \\
\hline Age & 0.352 & 0.061 & 0.374 & 0.039 \\
\hline Preoperative IOP & 0.246 & 0.246 & - & - \\
\hline Lens status & 0.445 & 0.016 & 0.163 & 0.346 \\
\hline Central corneal thickness & 0.395 & 0.034 & 0.261 & 0.095 \\
\hline Axial length & 0.047 & 0.809 & - & - \\
\hline Anterior chamber depth & 0.283 & 0.190 & - & - \\
\hline Postoperative follow-up period & -0.177 & 0.359 & - & - \\
\hline Preoperative central ECD & 0.465 & 0.011 & 0.313 & 0.056 \\
\hline Tube insertion level & 0.403 & 0.030 & 0.374 & 0.013 \\
\hline $\mathrm{TCD}$ in $\mathrm{N}$ & 0.176 & 0.362 & - & - \\
\hline $\mathrm{TCA}$ in $\mathrm{N}$ & -0.102 & 0.598 & - & - \\
\hline $\mathrm{TL}$ in $\mathrm{N}$ & 0.210 & 0.274 & - & - \\
\hline Change in TCD ( $\mathrm{N}$ vs. THT) & -0.214 & 0.264 & - & - \\
\hline Change in TCD ( $\mathrm{N}$ vs. NHT) & -0.268 & 0.160 & - & - \\
\hline Change in TCA ( $\mathrm{N}$ vs. THT) & 0.222 & 0.248 & - & - \\
\hline Change in TCA ( $\mathrm{N}$ vs. NHT) & -0.022 & 0.910 & - & - \\
\hline Change in TL ( $\mathrm{N}$ vs. THT) & -0.092 & 0.634 & - & - \\
\hline Change in TL ( $\mathrm{N}$ vs. NHT) & -0.007 & 0.971 & - & - \\
\hline
\end{tabular}

$\mathrm{IOP}=$ indicates intraocular pressure; $\mathrm{ECD}=$ endothelial cell density; TCD = tube-cornea distance; $\mathrm{N}=$ neutral head position; $\mathrm{TCA}=$ tube-cornea angle; TL = tube length; THT = temporalward head tilt; NHT = nasalward head tilt.

er, no association was found between the amount of ECD decrease and the head position-induced changes in the tube position. The univariate analysis showed that age, lens state, CCT, preoperative ECD, and the tube insertion level were associated with the change in central ECD after AGV implantation ( $p=0.061,0.016,0.034,0.011$, and 0.030 , respectively). The multivariate analysis showed that age and the tube insertion level significantly affected the change in ECD in the central corneal area after AGV implantation ( $p=0.039$ and 0.013 , respectively) (Table 4). With respect to the difference in ECD between the inferonasal and superotemporal corneal areas (postoperative inferonasal corneal ECD: $2,226.0 \pm 639.9$ cells $/ \mathrm{mm}^{2}$, postoperative superotemporal corneal ECD: 1,646.3 \pm 793.9 cells $/ \mathrm{mm}^{2}$ ), the postoperative follow-up period and tube insertion level were shown to be significant factors in both the univariate ( $p=0.004$ and 0.001 , respectively) and multivariate analyses ( $p=0.034$ and 0.007 , respectively) (Table 5).

\section{Discussion}

In the present study, we found that the intracameral position and length of the AGV tube may show some changes, albeit small, with mild alterations according to the head position in patients with glaucoma. Previously, a few studies have shown movement or migration of the intracameral tube of the GDDs [18-20]. In their case series, Law et al. [18] reported three eyes with $\mathrm{AGV}$ that had 3 to $4 \mathrm{~mm}$ of tube movement in the anterior chamber at different gazes. Park et al. [19] serially visualized AGV tube parameters using AS-OCT in 48 eyes over 12 months and found that tubes tended to shorten and migrate anteriorly, especially in eyes with a prior penetrating keratoplasty. Similarly, Tan et al. [20] also reported progressive anterior tube movement of Baerveldt drainage implants in a group where the tube was inserted freely in the anterior chamber. However, the effect of head tilt on the position or length of 
Table 5. Factors associated with differences in endothelial cell density between the inferonasal and superotemporal corneal area following Ahmed glaucoma valve implantation

\begin{tabular}{|c|c|c|c|c|}
\hline & \multicolumn{2}{|c|}{ Univariate analysis } & \multicolumn{2}{|c|}{ Multivariate analysis } \\
\hline & B coefficient & $p$-value & B coefficient & $p$-value \\
\hline Age & -0.078 & 0.713 & - & - \\
\hline Preoperative IOP & -0.064 & 0.768 & - & - \\
\hline Lens status & 0.143 & 0.496 & - & - \\
\hline Central corneal thickness & 0.160 & 0.446 & - & - \\
\hline Axial length & 0.210 & 0.313 & - & - \\
\hline Anterior chamber depth & 0.180 & 0.412 & - & - \\
\hline Postoperative follow-up period & 0.551 & 0.004 & 0.369 & 0.034 \\
\hline Preoperative central ECD & 0.117 & 0.578 & - & - \\
\hline Tube insertion level & 0.620 & 0.001 & 0.480 & 0.007 \\
\hline TCD in $\mathrm{N}$ & -0.198 & 0.342 & - & - \\
\hline $\mathrm{TCA}$ in $\mathrm{N}$ & -0.197 & 0.346 & - & - \\
\hline $\mathrm{TL}$ in $\mathrm{N}$ & -0.128 & 0.543 & - & - \\
\hline Change in TCD ( $\mathrm{N}$ vs. THT) & 0.147 & 0.483 & - & - \\
\hline Change in TCD ( $\mathrm{N}$ vs. NHT) & -0.075 & 0.722 & - & - \\
\hline Change in TCA ( $\mathrm{N}$ vs. THT) & 0.004 & 0.984 & - & - \\
\hline Change in TCA ( $\mathrm{N}$ vs. NHT) & -0.015 & 0.943 & - & - \\
\hline Change in TL ( $\mathrm{N}$ vs. THT) & 0.180 & 0.390 & - & - \\
\hline Change in TL ( $\mathrm{N}$ vs. NHT) & 0.054 & 0.798 & - & - \\
\hline
\end{tabular}

IOP = intraocular pressure; $\mathrm{ECD}=$ endothelial cell density; TCD = tube-cornea distance; $\mathrm{N}=$ neutral head position; TCA = tube-cornea angle; TL = tube length; THT = temporalward head tilt; NHT = nasalward head tilt.

the AGV tube has not been explored yet. To our knowledge, this is the first AS-OCT study to investigate the influence of different head positions on the intracameral tube position and length.

With regard to the mechanisms involved in the altered position or length of the tube, Law et al. [18] postulated that loosening of the nonabsorbable suture, extrusion of the suture from the sclera, and a relatively stronger adhesion of the fibrovascular capsule to the fornix compared with its adhesion to the sclera, or a combination caused such changes in the tube movement. However, they reported that such a dynamic tube movement was rare and noted only in 3 of 1,500 cases. In our study, AS-OCT detected smaller but significant changes in the tube position and length in different head positions of 29 eyes. The exact mechanism for these changes remains unclear. However, we speculate the following factors may be implicated. First, tilting of the head to one side may have induced changes in intracameral dimension of the eyeball, which may lead to possible alterations in the distance or angle between the ocular structure and tube. Our previous ASOCT study, although performed in different body postures, revealed differences in the anterior chamber angle parameters when compared between two different head positions [17]. Second, the head tilt-induced changes in tube parameters may be attributed to the ocular counter roll, where compensatory torsion of the eyeball occurs with a head tilt through contraction of the superior or inferior oblique muscle. This may pull the sclera and connective tissues around the plate of AGV, thereby influencing the intracameral position and/or length of the tube.

In addition to the position-induced alterations in tube parameters, we also found that these changes were not significantly associated with corneal endothelial cell loss following AGV surgery. In previous studies, endothelial cell loss after GDD implantation has already been reported 
with a few studies finding several risk factors for endothelial cell damage. Hau et al. [21] examined 40 eyes implanted with an aqueous shunt and found that the degree of peripheral anterior synechia was significantly associated with the lower corneal cell density. Other studies suggest that the jet of aqueous humor fluid near the tube, intermittent tube contact, and chronic inflammation caused by intermittent tube-uveal touch may affect endothelial cells $[12,13]$. In studies using AS-OCT, Koo et al. [15] revealed that tubes that are closer to the cornea lead to increased loss of adjacent endothelial cells. In contrast, Mendrinos et al. [16] showed no correlation between central or peripheral corneal endothelial cell loss and various tube parameters. In our study, although there were no factors significantly associated with central corneal endothelial cell loss including TCD, TCA, and TL and change of these tube parameters with head tilt, age and the tube insertion level appeared to be significant. Previously, Hau et al. [21] reported several risk factors associated with endothelial cell loss after GDD implantation; however, age was not included as a risk factor. Nevertheless, in their study, uveitic glaucoma was diagnosed in $65 \%$ of the individuals, unlike our study (neovascular glaucoma in 57\% of the patients); thus, inflammatory damage due to uveitis may have offset the effect of age. Moreover, corneal endothelial cells were found to be more vulnerable to mechanical damage at an advanced age following cataract surgery [22]. Similarly, corneal endothelial cells may be more vulnerable to mechanical loss at an older age after GDD implantation surgery.

Another notable observation of our study was that the level of tube entry in the anterior chamber was found to be associated with ECD loss. With similar values in the TCA, $\mathrm{TCD}$, or TL, the eyes with tubes inserted anterior to Schwalbe's line may be more susceptible to corneal endothelial cell damage than those with tubes inserted at the level of or posterior to Schwalbe's line. Fine movements of the tube, occurring with eyeball movements, are more likely to readily injure the corneal endothelial cells, which have migrated toward the superotemporal quadrant to replace the damaged ones. This is supported by our finding that the tube entry level was a risk factor significantly associated with the difference between values of superotemporal ECD and inferonasal ECD. The postoperative follow-up period was also significantly associated with the difference between values of superotemporal ECD and inferonasal ECD. This significant difference may be due to sustained damage to the superotemporal ECD by the tube.

There are some limitations in the current study. First, the sample size of the present study was small. Second, an unidentical length of postoperative follow-up periods among study patients limited extrapolation of our findings. Third, the lens status was not identical among the study patients, which could have affected the TCA or changes in TCA. Fourth, measurement of tube-related parameters was performed manually from the AS-OCT images without automatic tracking. One may claim that it is unclear whether the scan line passing along the tube during AS-OCT scanning has been fitted to the line at another scanning while head-tilting. However, we used the guide image in the image analysis report with the front eye image and scanning line to ensure that the scan line passing along the tube during AS-OCT scanning fitted to the line at another scanning while head-tilting. We selected and used the images for analysis only when the scanning line was parallel to the center of the tube. Fifth, the present study only investigated the effect of mild alterations of head positions (lateral tilts up to $30^{\circ}$ ). The magnitude of changes in tube positions induced by head or body positions may be greater in recumbent body postures such as prone or lateral decubitus positions.

In conclusion, although mild alterations of head positions induced changes in the intracameral tube position of glaucoma drainage implants, it did not significantly affect postoperative ECD loss. However, eyes with tubes inserted anteriorly to Schwalbe's line may be more susceptible to corneal endothelial cell damage. Further studies are required to address whether a greater range of positional alterations lead to changes in tube position, which may significantly affect the corneal endothelial cells.

\section{Conflict of Interest}

No potential conflict of interest relevant to this article was reported.

\section{References}

1. Stamper RL, Lieberman MF, Drake MV. Medical treatment of glaucoma: general principles. In: Stamper RL, Lieberman MF, Drake MV. Becker-Shaffer's diagnosis and 
therapy of the glaucomas. 8th ed. Philadelphia: Mosby; 2009. p. 346.

2. Mosaed S, Minckler DS. Aqueous shunts in the treatment of glaucoma. Expert Rev Med Devices 2010;7:661-6.

3. Francis BA, Singh K, Lin SC, et al. Novel glaucoma procedures: a report by the American Academy of Ophthalmology. Ophthalmology 2011;118:1466-80.

4. Saheb H, Ahmed II. Micro-invasive glaucoma surgery: current perspectives and future directions. Curr Opin Ophthalmol 2012;23:96-104.

5. Bovee CE, Pasquale LR. Evolving surgical interventions in the treatment of glaucoma. Semin Ophthalmol 2017;32:91-5.

6. Lavia C, Dallorto L, Maule M, et al. Minimally-invasive glaucoma surgeries (MIGS) for open angle glaucoma: A systematic review and meta-analysis. PLoS One 2017;12:e0183142.

7. Gedde SJ, Schiffman JC, Feuer WJ, et al. Treatment outcomes in the Tube Versus Trabeculectomy (TVT) study after five years of follow-up. Am J Ophthalmol 2012;153:789803.

8. Gedde SJ, Feuer WJ, Shi W, et al. Treatment outcomes in the primary tube versus trabeculectomy study after 1 year of follow-up. Ophthalmology 2018;125:650-63.

9. Khanna CL, Mahr MA, Hodge DO, et al. Variations in trabeculectomy and glaucoma drainage device use for the treatment of glaucoma in the United States. Ophthalmol Glaucoma 2018;1:139-43.

10. Wilson MR, Mendis U, Paliwal A, Haynatzka V. Longterm follow-up of primary glaucoma surgery with Ahmed glaucoma valve implant versus trabeculectomy. Am J Ophthalmol 2003;136:464-70.

11. Kim KN, Lee SB, Lee YH, et al. Changes in corneal endothelial cell density and the cumulative risk of corneal decompensation after Ahmed glaucoma valve implantation. Br J Ophthalmol 2016;100:933-8.

12. Topouzis F, Coleman AL, Choplin N, et al. Follow-up of the original cohort with the Ahmed glaucoma valve implant. Am J Ophthalmol 1999;128:198-204.

13. McDermott ML, Swendris RP, Shin DH, et al. Corneal endothelial cell counts after Molteno implantation. Am J Ophthalmol 1993;115:93-6.

14. Oh WH, Kim TW, Park KH, Kim DM. Location of the tube tip in the anterior chamber and change in corneal endothelium after Ahmed valve implantation. J Korean Ophthalmol Soc 2013;54:469-74.

15. Koo EB, Hou J, Han Y, et al. Effect of glaucoma tube shunt parameters on cornea endothelial cells in patients with Ahmed valve implants. Cornea 2015;34:37-41.

16. Mendrinos E, Dosso A, Sommerhalder J, Shaarawy T. Coupling of HRT II and AS-OCT to evaluate corneal endothelial cell loss and in vivo visualization of the Ahmed glaucoma valve implant. Eye (Lond) 2009;23:1836-44.

17. Park JH, Yeon DY, Yoo C, Kim YY. Effect of lateral decubitus body posture on anterior chamber angle in healthy subjects: an anterior segment optical coherence tomography study. J Glaucoma 2017;26:608-12.

18. Law SK, Coleman AL, Caprioli J. Dynamic tube movement of Ahmed glaucoma valve. J Glaucoma 2009;18:62831.

19. Park HY, Jung KI, Park CK. Serial intracameral visualization of the Ahmed glaucoma valve tube by anterior segment optical coherence tomography. Eye (Lond) 2012;26:1256-62.

20. Tan AN, De Witte PM, Webers CA, et al. Baerveldt drainage tube motility in the anterior chamber. Eur J Ophthalmol 2014;24:364-70.

21. Hau S, Scott A, Bunce C, Barton K. Corneal endothelial morphology in eyes implanted with anterior chamber aqueous shunts. Cornea 2011;30:50-5.

22. Cho YK, Chang HS, Kim MS. Risk factors for endothelial cell loss after phacoemulsification: comparison in different anterior chamber depth groups. Korean J Ophthalmol 2010;24:10-5. 